\title{
Development of Multiscale Biological Image Data Analysis: Review of 2006 International Workshop on Multiscale Biological Imaging, Data Mining and Informatics, Santa Barbara, USA (BII06) Manfred Auer ${ }^{\dagger 1}$, Hanchuan Peng ${ }^{* \dagger 2}$ and Ambuj Singh ${ }^{\dagger 3}$
}

Address: ${ }^{1}$ Life Sciences Division, Lawrence Berkeley National Laboratory, Berkeley, CA, USA, ${ }^{2}$ Janelia Farm Research Campus, Howard Hughes Medical Institute, Ashburn, VA, USA and ${ }^{3}$ Department of Computer Science, University of California, Santa Barbara, CA, USA

Email: Manfred Auer - mauer@lbl.gov; Hanchuan Peng* - pengh@janelia.hhmi.org; Ambuj Singh - ambuj@cs.ucsb.edu

* Corresponding author †Equal contributors

from 2006 International Workshop on Multiscale Biological Imaging, Data Mining and Informatics

Santa Barbara, CA, USA. 7-8 September 2006

Published: 10 July 2007

BMC Cell Biology 2007, 8(Suppl I):SI doi:I0.II86/I47I-2I2I-8-SI-SI

This article is available from: http://www.biomedcentral.com/|47|-2|2I/8/SI/SI

(C) 2007 Auer et al; licensee BioMed Central Ltd.

This is an open access article distributed under the terms of the Creative Commons Attribution License (http://creativecommons.org/licenses/by/2.0), which permits unrestricted use, distribution, and reproduction in any medium, provided the original work is properly cited.

\begin{abstract}
The 2006 International Workshop on Multiscale Biological Imaging, Data Mining and Informatics was held at Santa Barbara, on Sept 7-8, 2006. Based on the presentations at the workshop, we selected and compiled this collection of research articles related to novel algorithms and enabling techniques for bio- and biomedical image analysis, mining, visualization, and biology applications.
\end{abstract}

\section{Introduction}

With the development of advanced imaging techniques, the number of biological images (e.g. cellular and molecular images, as well as medical images) acquired in digital forms is growing rapidly. Large-scale bioimage databases are becoming available. Analyzing these images has been proven critical for biologists to seek answers to many biological problems. Novel techniques that enable millimeter-, micrometer- and nanometer-scale observations of the same specimen are also emerging. The potential of mining the information in bioimages, especially at different scales of resolution and complexity, is enormous for a deeper understanding of physiology and pathogenesis, for basic sciences as well as for applied sciences and bioengineering. We organized the 2006 International Workshop on Multiscale Biological Imaging, Data Mining and Informatics (BII06) at Santa Barbara, CA, USA, on Sept 7-8, 2006 [1]. It was a follow-up event of the 2005 International
Workshop on Bioimage Informatics held at Stanford University [2].

BII06 succeeded in bringing together interdisciplinary researchers to identify problems at each level of imaging and particularly across different imaging modalities/ scales, and present their answers using cutting edge image data analysis, computer vision, data mining, machine learning, visualization, and informatics methods. Over 90 people, including 30 faculty members, more than 30 postdoctoral scholars and graduate students, and other scientists from various research institutes, attended the workshop. There were 13 invited talks, 16 peer-reviewed talks, and 14 peer-reviewed posters. The program concluded with a panel discussion that allowed interdisciplinary experts to brainstorm the challenges for effective mining of the increasingly complex bioimage data. All sessions were very interactive. There were a number of ques- 
tions from the audience and the discussions spilled over into coffee and meal breaks. Short abstracts for the invited talks and two-page papers for all peer-reviewed talks and posters were published in a printed proceedings, which is freely available on the workshop website. Besides the research talks, posters, and the panel, four vendors had product exhibitions at the workshop. Three of them delivered short oral presentations during the lunch hour.

\section{Challenges of bioimage informatics}

Besides reporting a number of exciting bioimaging and image informatics projects, the workshop attendees had an extensive discussion of the following challenges.

\section{- The demand for bioimage informatics techniques}

To biologists, a way to organize and share the large amount of images and search them using metadata or image features is very important. Biologists were of the opinion that image registration and mosaicing are very important image processing tasks. Modeling of processes, at different levels and different resolutions, in order to classify and predict different biological entities and processes is absolutely critical. High-resolution displays provide an opportunity for interactive exploration of data; however, browsing through multiple monitors can be challenging.

\section{- The need of multiscale imaging}

The data from even a single image is huge and we have not yet been able to extract all the information from it. The current deluge of images only exacerbates the challenges. Given the constraints of time and money, is it really worthwhile to spend energy on obtaining multiscale images? Similar questions were raised about highthroughput imaging - is it achievable, is it desirable, is automation the answer? A number of people were of the opinion that as much imaging information should be collected as possible, even though this information cannot be analyzed in depth at this point.

\section{- Collaboration and communication between biologists and engineers}

While biologists and engineers have been using many similar terminologies with distinct meanings (such as "labeling"), it seems that the collaboration and communication between different fields are not going to happen naturally and that it is critical to force the mixture. Both groups of scientists would benefit from this. Tweaking with a microscope for 5 minutes could save tweaking the parameters of a computer algorithm for a few months. Further, data sharing and knowledge sharing should be on a common platform - programs written by computer scientists to be used by biologists should be user-friendly and data provided by biologists should be as complete as possible.

\section{- Common bioimage informatics problems and bench test data sets}

For the image processing and analysis community, four specific problems were identified as representative: (1) segmentation, (2) connections in space or time, (3) registration or atlas building, and (4) classification. There is a need to provide a small set of biological datasets with ground truth and a small set of image processing tools that anybody can use and that provides a benchmark for any new algorithms.

\section{- Modeling}

The goal of science is to make realistic models of what is happening in nature and often one very important component of realizing those models is biological intuition. There is a need to deal with all the data that can be acquired. The hope is to catalog problems and solutions such that after 15-20 years, the ad-hoc pieces will be integrated together and science can progress. Models should be neither too complex, nor too trivial, to advance the understanding of biology. This raises some fundamental questions, e.g. what to abstract, how to abstract, and how much to abstract.

\section{Selected papers}

We solicited full-paper submissions from the workshop participants who had talk presentations. Each submission was peer-reviewed by at least two reviewers. We accepted 9 papers and compiled this supplement of BMC Cell Biology, an online open access journal. We hope the freeavailability of these papers can maximize their visibility.

The contents of these papers include new image analysis and mining algorithms, data visualization, biological applications, enabling supercomputing techniques, and computer vision and machine learning methods to solve other biology problems. In summary, Maree et al [3] developed a cell image classification method based on random subwindows and random trees. Long et al [4] presented a phenotype clustering analysis for breast epithelial cells, based on the 3D nuclear protein distributions. Altinok et al [5] presented a method to extract dynamics information from time-lapse live cell microtubule images. Cecchi et al [6] developed a method to extract correlation relationships from brain functional MRI data where there are tens of thousands of variables. Singh [7] presented a new molecule retrieval method based on the similarity of molecule surface information such as shape, field strength and superposition. Peng et al [8] developed a suite of techniques to analyze the in situ gene expression patterns of fly embryogenesis, and have applied their methods to detecting regulatory motifs of gene sequences and automation of gene expression pattern annotation using anatomical ontology vocabularies. Boucheron et al [9] conducted a comparative analysis of both the multispectral and RGB 
histopathology images, and found a minimal improvement of class prediction accuracy by simply increasing the spectral bands of imaging. Rao et al [10] presents a highperformance computing solution to handle image data sets at the gigabyte level, by decomposing $3 \mathrm{D}$ image as small segments that are assigned to unique processors of the 3D torus architecture of the IBM Blue Gene/L machine. Staadt et al [11] summarized their work on interactive processing and visualization of image data for protein surface, retinal optical coherence tomographic data, and gene expression images of early stage fly embryogenesis.

\section{Acknowledgements}

We thank the referees for their dedication and effort in reviewing the manuscripts submitted for this supplement. We thank the Bll06 workshop program committee for help in organization of the workshop, and the UC Discovery Grant and the Center for Bioimage Informatics, UC Santa Barbara, for sponsorship of the workshop. We also thank the BMC Editorial Office for their support and encouragement in making this issue possible.

This article has been published as part of BMC Cell Biology Volume 8 Supplement I, 2007: 2006 International Workshop on Multiscale Biological Imaging, Data Mining and Informatics. The full contents of the supplement are available online at http://www.biomedcentral.com/|47|-2|2|/8?issue=SI

\section{References}

I. [http://www.bioimageinformatics.org/2006].

2. [http://www.bioimageinformatics.org/2005].

3. Maree R, Geurts P, Wehenkel L: Random subwindows and extremely randomized trees for image classification in cell biology. BMC Cell Biology 2007, 8(Suppl I):S2.

4. Long FH, Peng HC, Sudar D, Lelièvre SA, Knowles DW: Phenotype clustering of breast epithelial cells in confocal images based on nuclear protein distribution analysis. BMC Cell Biology 2007, 8(Suppl I):S3.

5. Altinok A, Kiris E, Peck AJ, Feinstein SC, Wilson L, Manjunath BS, Rose K: Model based dynamics analysis in live cell microtubule images. BMC Cell Biology 2007, 8(SuppI I):S4.

6. Cecchi GA, Rao AR, Centeno MV, Baliki M, Apkarian AV, Chialvo DR: Identifying directed links in large scale functional networks: application to brain fMRI. BMC Cell Biology 2007, 8(SuppI I):S5.

7. Singh R: Surface similarity-based molecular query-retrieval. BMC Cell Biology 2007, 8(SuppI I):S6.

8. Peng HC, Long FH, Zhou J, Leung G, Eisen MB, Myers EW: Automatic image analysis for gene expression patterns of fly embryos. BMC Cell Biology 2007, 8(SuppI I):S7.

9. Boucheron LE, Bi ZQ, Harvey NR, Manjunath BS, Rimm DL: Utility of multispectral imaging for nuclear classification of routine clinical histopathology imagery. BMC Cell Biology 2007, 8(Suppl I):S8.

10. Rao AR, Cecchi GA, Magnasco M: High performance computing environment for multidimensional image analysis. $B M C$ Cell Biology 2007, 8(SuppI I):S9.

II. Staadt OG, Natarajan V, Weber GH, Wiley DF, Hamann B: Interactive processing and visualization of image data for biomedical and life science applications. BMC Cell Biology 2007, 8(Suppl I):SIO.

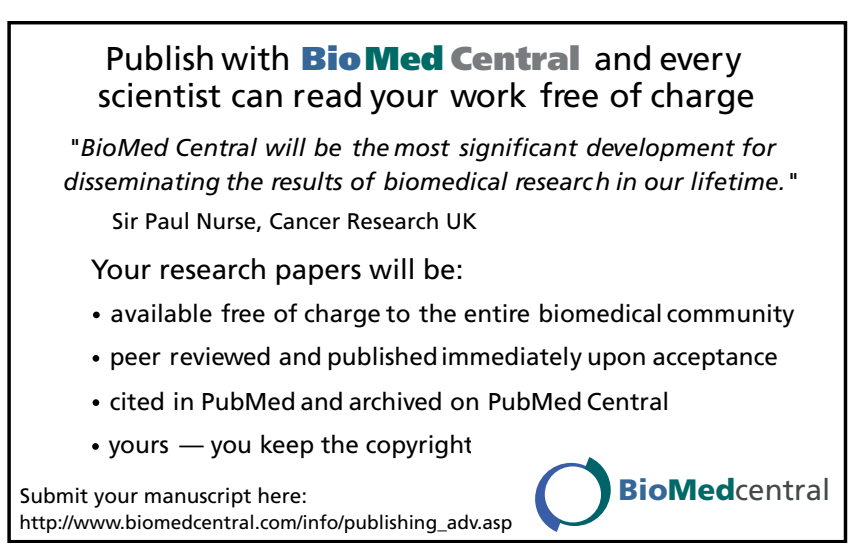

